\title{
Parallelization of Karcsmarz Algorithm using Graphic Cards for Seismic Imaging
}

\author{
Muhamad Ilyas $^{1}$, Agah Drajat Garnadi ${ }^{1} \&$ Sri Nurdiati $^{1}$
}

Department of Mathematics, Bogor Agricultural University, Bogor, Indonesia 16680

Email: reiken7@gmail.com, agah.garnadi@gmail.com, s.nurdiati@gmail.com

\begin{abstract}
We present a SCILAB implementation of algebraic iterative reconstruction methods for discretizations of inverse problems in seismic imaging. These so-called row action methods rely on semi-convergence for achieving the necessary regularization of the problem. We parallelize this method using NVIDIA graphics cards and SCILAB toolbox. We provide a few simplified test problems in seismic imaging to test those solver.
\end{abstract}

16 Keywords: Algebraic reconstruction methods, Parallelization, Seismic imaging, 17 Systems of linear equations.

\section{Introduction}

19 Iterative reconstruction method for computing solutions to discretization of 20 inverse problems have been used for decades in medical imaging, geophysics, 21 material science and many other disciplines that involve 2D and 3D imaging 22 [1]. This paper presents algebraic reconstruction techniques to solve large linear 23 systems of the form

$$
A x \cong b, A \in \mathbb{R}^{m \times n}
$$

25 used in tomography and many other inverse problems. We assume that the 26 elements of matrix $A$ are nonnegative and contains no zero rows or columns.

27 There are no restriction on the dimensions.

28 We can write the system of linear equations with $\mathrm{M}$ equations with $\mathrm{N}$ unknown 29 variables: 
$30 \quad \mathbf{a}_{i}^{T} \mathbf{x}=b_{i}, \quad i=1,2, \ldots, M$

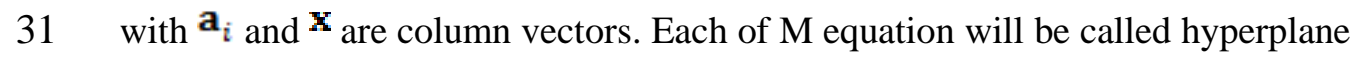

32 in N-dimension Euclidean space, $\mathbb{R}^{N}$.

\section{Parallel Computing}

34 The idea of parallel computing is to share a calculation to some processing unit

35 and calculate it in each processing unit independently. The restriction of this

36 technique is the fully sequential method, for example a calculation that based on

37 previous calculation. In this case, it's based on programmer judgment when and

38 where parallel computing will be used.

39 Parallel computing used in this paper based on NVIDIA GPU. NVIDIA is well-

40 known graphic cards manufacturer and with its most recent innovation, graphic

41 processing unit (GPU) which can be accessed by CUDA (Compute Unified

42 Device Architecture), programmer can easily use GPU to calculate some

43 mathematical problems. Programming language used in CUDA are similar to

$44 \mathrm{C} / \mathrm{C}++$ with some specific instruction to access the task to processing unit.

45 Parallel computing with CUDA could be used with sciGPGPU package, a

46 toolbox in SCILAB. With this package, programmer can access CUDA

47 commands along with SCILAB command, which makes calculation easier and

48 more flexible.

\section{$493 \quad$ Algebraic Reconstruction Techniques}

50 The Algebraic Reconstruction Technique (ART) is one of the method

51 used in commercial machine. This iterative method presented by S.

52 Kaczmarz in 1937 in his paper "Angenäherte auflösung von systemen

53 linearer gleichungen". 
54 The core of ART method is the row-action method that treat the equation 55 one at a time during the iterations. ART method find orthogonal 56 projection $\mathbf{x}_{p}$ from $\mathbf{x}^{*}$ to the hyperplane $\mathbf{a}_{k}^{T} \mathbf{x}=b_{k}$ for $k=1,2, \ldots, M$. For 57 each iteration, $\mathbf{x}_{p}$ will convergent to the solution if the vectors $\mathbf{a}_{1}, \mathbf{a}_{2}, \ldots$, $58 \mathbf{a}_{M}$ span $\mathbb{R}^{N}$. Therefore, in each iteration, we calculate the iterative 59 vector:

60

$$
\mathbf{x}_{p}=\mathbf{x}^{*}+\frac{\mathbf{b}-\mathbf{a}_{k}^{T} \mathbf{x}}{\mathbf{a}_{k}^{T} \mathbf{a}_{k}} \mathbf{a}_{k}
$$

\section{Seismic Imaging}

62 Seismic tomography is a technique for imaging earth sub surface characteristics.

63 We placed the sources on the right side of the area and the receivers on the left

64 side and the surface of the area. The imaging burst a ray from each sources to 65 every receivers on observation area. This ray pierces the observation area and 66 received as a series of number. The output from the experiment is a series of 67 numbers listed in vector form which is a value from each source and receiver 68 pairwise. Our problem is we have to look for earth sub surface form and 69 characteristic from given matrix and the location of ray sources and receivers.

70 Below is the figure for the illustration of seismic tomography. In this 71 images, green point indicates sources andblue point indicates receivers. 


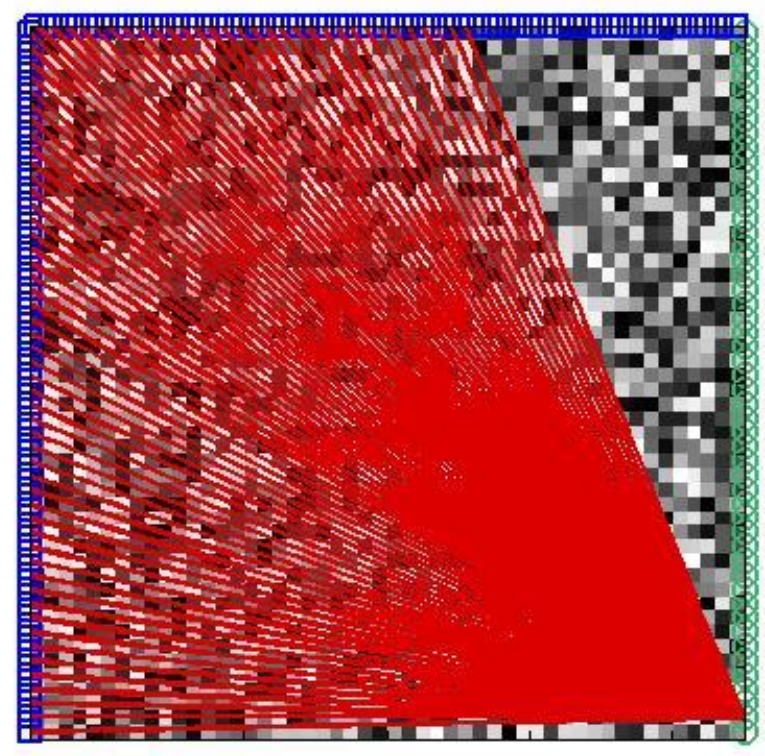

73

74

75

Figure 1 Illustration of seismic tomography.

76 We will also present the images of our seismic tomography model problem

77 known as tectonic phantom model. We need this model as the value in $x$ matrix

78 for ART problems. 


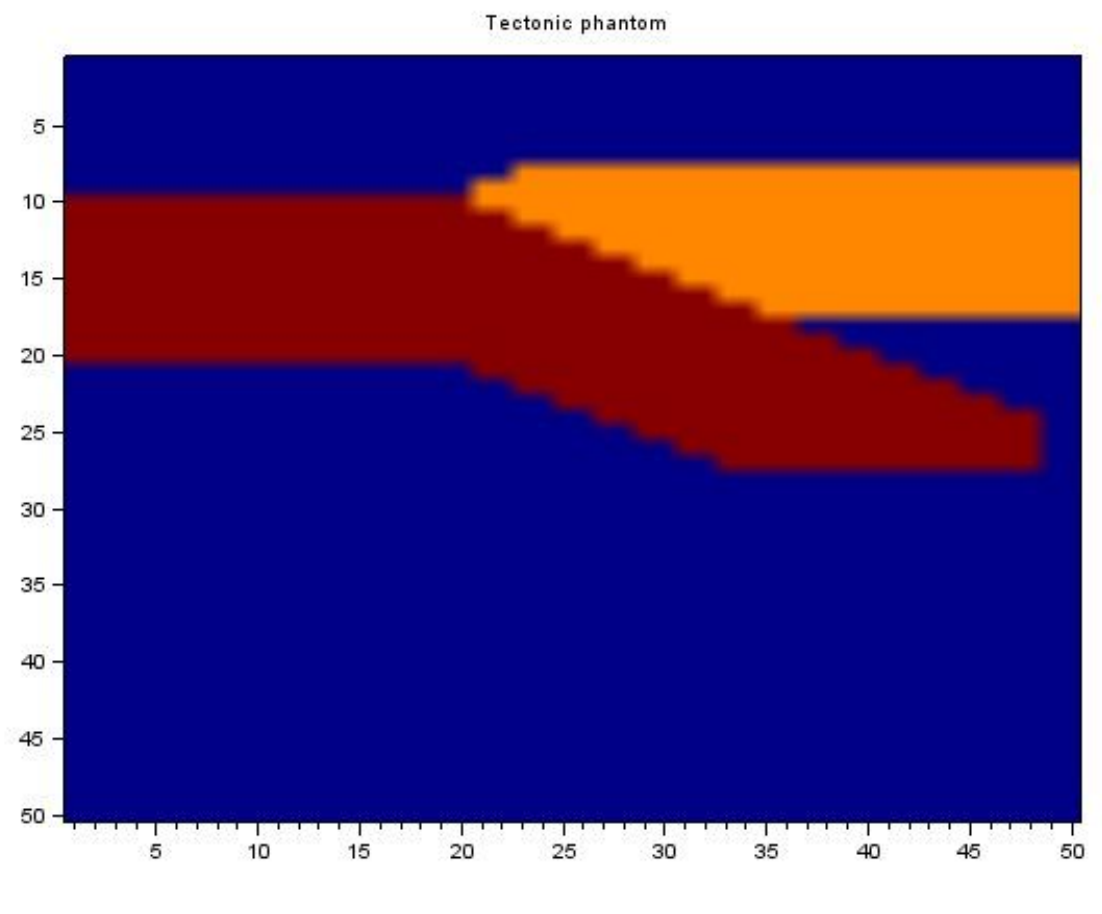

82 Characteristics of matrix used in experiment is given below.

83

84

Table 1 MATRIX CHARACTERISTICS

\begin{tabular}{|c|c|}
\hline & Seismic tomography \\
\hline Size of Coefficient Matrix & 5000 x 2500 \\
\hline Number of nonzero elements of Coefficient Matrix & $288400(2.31 \%)$ \\
\hline Maximum Value of Coefficient Matrix & 1.4001 \\
\hline Minimum Value of Coefficient Matrix & 0.0072 \\
\hline Maximum Value of RHS Vector & 51.0157 \\
\hline Minimum Value of RHS Vector & 0 \\
\hline
\end{tabular}

85 
86 These seismic tomography coefficient matrix is based on AIR Tools - A

87 MATLAB package of algebraic iterative reconstruction methods [1]. By

88 multiply the desired coefficient matrix with the seismic imaging model

89 problem.

\section{Computational Result}

91 All the computational experiment in this paper is conducted in computer

92 laboratory, Department of Mathematics, Faculty of Mathematics and Natural

93 Sciences, Bogor Agricultural University. Computer specifications used are:

94 Processor Intel Core i5-3317U 1.7 GHz, RAM 4 GB, VGA NVIDIA GeForce

95 GT 635M. Experiment done in Ubuntu OS 10.10 with sciGPGPU 1.3, SCILAB

$96 \quad 5.4 .0$ and CUDA 4.0.

97 Algebraic reconstruction techniques (ART) produces an iterative solution with

98 given coefficient matrix and right hand side vector. We reproduce approximate

99 solution with increased iteration numbers by using original coefficient matrix

100 and right hand side vector. Both tomography techniques produces different

101 results below.

102 Additionally, we test the problems by giving some perturbation to the right hand

103 side vector in the form of random error vector. With an additional vector, we

104 also reproduce approximate solution with increased iteration numbers.

105

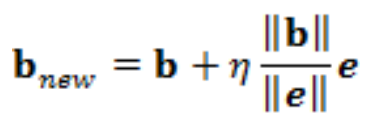

106 where $\boldsymbol{e}$ is a random error vector and $\eta$ is some small number (in this

107 experiment we use $\eta=0.05$ ). 
108 Below are approximate solution to the seismic tomography problems with

109 increased ART iterations, with original and perturbed right hand side vector.

110
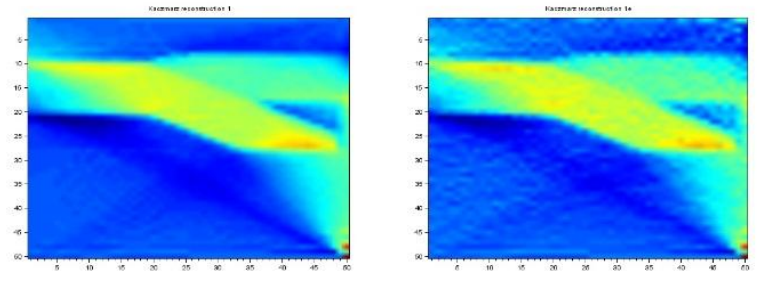

\begin{tabular}{|l|l|}
\hline 1 ART iteration (original) & 1 ART iteration (perturbed) \\
\hline$\left\|A x^{*}-b\right\|=1002.028$ & $\left\|A x^{*}-b\right\|=1016.642$ \\
\hline Time $=0.608 \mathrm{~s}$ & Time $=0.749 \mathrm{~s}$ \\
\hline
\end{tabular}

111

112
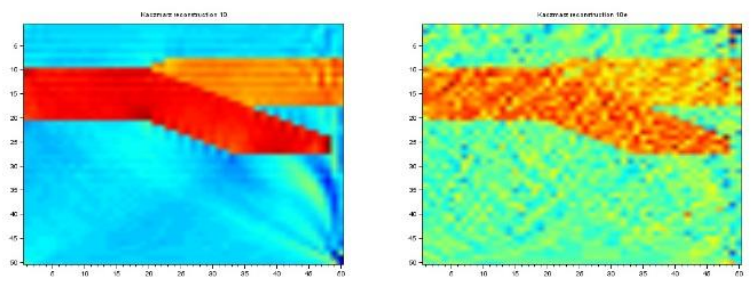

\begin{tabular}{|l|l|}
\hline 10 ART iteration (original) & $\begin{array}{l}10 \quad \text { ART iteration } \\
\text { (perturbed) }\end{array}$ \\
\hline$\left\|A x^{*}-b\right\|=54.019$ & $\left\|A x^{*}-b\right\|=97.599$ \\
\hline Time $=4.898 \mathrm{~s}$ & Time $=3.744 \mathrm{~s}$ \\
\hline
\end{tabular}

113 
114
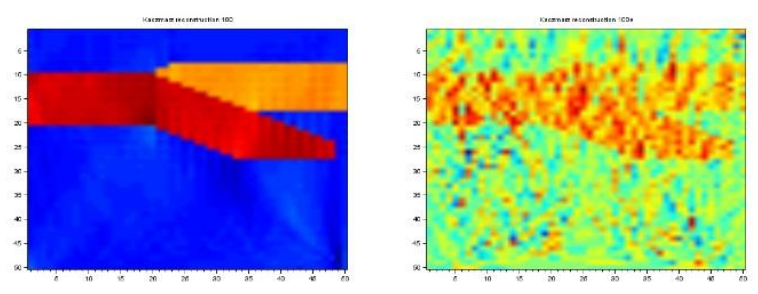

\begin{tabular}{|l|l|}
\hline $\begin{array}{l}100 \quad \text { ART iteration } \\
\text { (original) }\end{array}$ & $\begin{array}{l}100 \quad \text { ART iteration } \\
\text { (perturbed) }\end{array}$ \\
\hline$\left\|A x^{*}-b\right\|=1.387$ & $\left\|A x^{*}-b\right\|=79.016$ \\
\hline Time $=33.166 \mathrm{~s}$ & Time $=37.331 \mathrm{~s}$ \\
\hline
\end{tabular}

115

116
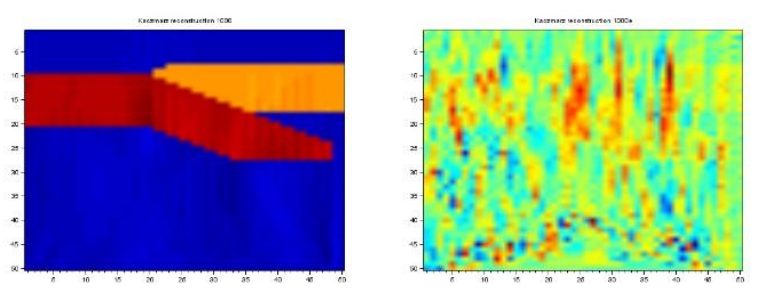

\begin{tabular}{|l|l|}
\hline $\begin{array}{l}1000 \quad \text { ART iteration } \\
\text { (original) }\end{array}$ & $\begin{array}{l}1000 \quad \text { ART iteration } \\
\text { (perturbed) }\end{array}$ \\
\hline$\left\|A x^{*}-b\right\|=0.1688$ & $\left\|A x^{*}-b\right\|=77.796$ \\
\hline Time $=385.447 \mathrm{~s}$ & Time $=386.243 \mathrm{~s}$ \\
\hline
\end{tabular}

117

118 From above result for seismic tomography, by additional ART iterations,

119 approximate solutions is closer to exact solution, shown by smaller value of 
$120\left\|A x^{*}-b\right\|$. This is also matched with approximation data resized to matrix

121 form and represented by scaled image.

122 On the other hand, perturbed right hand side also give approximate solution that

123 is closer to exact solution, shown by smaller value of $\left\|A x^{*}-b\right\|$, even with

124 slower rate of convergence.The more ART iterations performed, approximation

125 data represented by scaled images is worse than small ART iterations. As we

126 could see that given some random normal perturbation matrix, the images is

127 blur and can't be recognized as a tectonic phantom model.

\section{$128 \quad 6 \quad$ Conclusion}

129 Seismic tomography are widely used in earth sub surface imaging. This

130 experiment shows that the parallelization of algebraic reconstruction techniques 131 to solve system of linear equations is successful. By adding more ART

132 iterations, approximate solutions is closer to exact solution shown by smaller

133 value of norm $\left\|A x^{*}-b\right\|$, which is also matched with approximation data that

134 represented by scaled image.

135 With an additional error vector in hand right side vector, which we called 136 perturbed systems, the approximate solution is harder to converge, shown by the

137 value of norm $\left\|A x^{*}-b\right\|$. In the end, even with increased ART iteration, the 138 scaled image produced by perturbed system become more blurred and can't be 139 recognized as a tectonic phantom model, even though time elapsed for solving 140 both equation is not significantly different.

\section{Acknowledgement}

142 This work was funded by the Ministry of Education and Culture, Republic of 143 Indonesia, through the BOPTN Fiscal Year 2013, with contract no: 144 232/IT3.41.2/L2/SPK/2013. 


\section{$\begin{array}{lll}145 & 7 & \text { Nomenclature (if necessary) }\end{array}$}

146 List the nomenclature in alphabetical order. List Roman letters followed by

147 Greek symbols followed by subscript and superscripts.

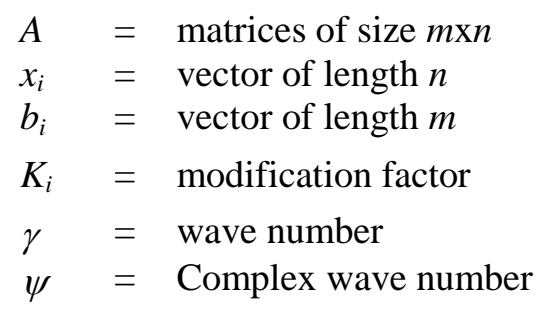

\section{References}

150 [1] Hansen, P.C \& Saxild-Hansen, M., AIR Tools - A MATLAB package of 151 algebraic iterative reconstruction methods. Journal of Computational and Applied Mathematics, 2012.

[2] sciGPGPU QuickStart, http://forge.scilab.org/index.php/p/sciCuda.

[3] Grama, A., Gupta A., Karypis, G., \& Kumar, V., Introduction to Parallel Computing, London: Addison Wesley, 2003.

[4] Kaczmarz, S. 1937. Angenäherte Auflösung von Systemen linearer Gleichungen, Bulletin de l'Acad' emie Polonaise des Sciences et Lettres, A35 (1937), pp. 355-357.

\section{$1598 \quad$ Manuscript Content}

160 The contents of the paper should be in the following order:

161 1. Title of Paper

162 2. Author names and affiliation

163 3. Abstract

164 4. Body of the text (Introduction

165 5. Acknowledgements

166 6. Nomenclature

167 7. References 